\title{
Plasticizer-induced changes in the mechanical rate of response of film coatings: an approach to quantitating plasticizer effectiveness
}

\author{
Christopher M. Sinko and Gordon L. Amidon \\ College of Pharmacy, University of Michigan, Ann Arbor, MI 48109-1065 (U.S.A.)
}

(Received 14 November 1988)

(Modified version received 24 April 1989)

(Accepted 28 April 1989)

Key words: Creep compliance; Plasticizing efficiency; Retardation spectrum;

Time-temperature superposition; Free volume; Film coating

\section{Summary}

The plasticization of Eudragit S100, a polymeric-based enteric coating, with polyethylene glycol 200 and dibutyl phthalate was investigated by analyzing changes in the time scale of mechanical response. Since all polymeric film coating solutions pass through the rubber-to-glass transition as solvent evaporates, this study focuses on the plasticization-induced changes in the rate of mechanical response in this region. Solvent cast films were mechanically analyzed using a low strain clongational creep compliance procedure and intrinsic mechanical response was analyzed via the application of a phenomenological approach of retardation spectrum analysis. Results indicate that at constant temperature, the addition of plasticizer changes the time scale of response by shifting the spectra negatively within the experimental time window. Using a free volume analysis, a plasticizing effectiveness term, $\beta$, was calculated for the plasticizers in this study with $\beta_{\mathrm{DBP}}=0.144$ and $\beta_{\mathrm{PEG}}=0.108$. The higher value of $\beta$ indicates a greater propensity to relax stress in the rubber-to-glass transition. The application of this technique may yield more relevant mechanical information for film coating systems without relying solely on glass transition temperature changes.

\section{Introduction}

With the advent of efficient film coating techniques in the past 15 years, formulators have turned their attention towards polymeric-based coating systems. From a controlled release perspective, it is critically important to reduce the incidence of macroscopic flaws such as cracks in order to obtain optimal performance of the particular dosage form. Cohesive and adhesive failure in a polymeric film coated dosage form are gener-

Correspondence: C.M. Sinko, College of Pharmacy, The University of Michigan, Ann Arbor, MI 48109-1065, U.S.A. ally caused by the level of residual internal stress that is generated during the film forming process (Croll, 1979).

Maintaining a high rate of relaxation is critical in a film-forming system because solvent loss sets a bulk strain in the material which, as a continuum, will introduce stress as well. Solvent loss will also slow the configurational changes that cause the relaxation of a polymeric material until, at a given concentration, the system behaves like a solid. This reduction in the rate of relaxation occurs in the primary softening dispersion or the glass transition region of the polymer. Further loss of solvent will generate stress at a greater rate than can be relaxed thus resulting in a net increase 
in internal stress which could ultimately lead to premature failure of the coating.

The addition of a plasticizer will lower the critical concentration of vehicle required to maintain a high rate of response at a particular temperature. For example, Croll (1979) found that at $25^{\circ} \mathrm{C}$, the concentration of toluene must be $16 \%$ or greater in order for polystyrene to be rubbery. If a good plasticizer such as tricresyl phosphate were added to the system, the critical concentration would be appreciably lower.

Concentration and temperature effects on the viscoelastic response of polymeric materials have been well documented (Ferry, 1980a and b). In an effort to isolate plasticizer-induced effects on viscoelastic response in the primary softening dispersion, we elected to evaluate properties of dry films at elevated temperatures in the glass transition region rather than at specific concentrations of the vehicle in order to avoid complications due to solvent loss. This technique, which has been previously presented (Sinko and Amidon, 1987) and now described in more detail, allows us to characterize the primary softening dispersion and to describe intrinsic mechanical response using a phenomenological approach of retardation spectrum analysis. In this study, the effects of the plasticizers, dibutyl phthalate and polyethylene glycol 200, on the viscoelastic properties of Eudragit S100, a copolymer consisting of polymethyl methacrylate and polymethacrylic acid, are measured by evaluating shifts of the retardation spectra within an experimental time window.

\section{Materials and Methods}

\section{Film preparation}

Eudragit S100 (Rohm Pharma GMBH, Weiterstadt, F.R.G. lot no. 05.80023) was obtained as a powder and used as received. Ten percent $w / w$ solutions of the polymer were prepared in analytical grade isopropyl alcohol. Polyethylene glycol 200 (PEG) (Sigma Chemical Co., St. Louis, MO; lot no. $84 \mathrm{~F}-0605$ ) was added to the solutions at concentrations of $5-25 \%$ by weight of the polymer. Dibutyl phthalate (DBP) (Sigma Chemical Co., St. Louis, MO; lot no. 114F-3447) was added to the solutions at concentrations of $10-30 \%$ by weight of the polymer. Two milliliters of each solution was poured onto a glass plate and placed in an oven at $35^{\circ} \mathrm{C}$ for $45 \mathrm{~min}$. The films were lifted carefully using a razor blade and cut into $15 \times 2.5 \mathrm{~mm}$ strips. Each strip was placed into a separate vial and dried to constant weight at $50^{\circ} \mathrm{C}$ and stored in a dessicator over anhydrous calcium sulfate for 2 weeks.

\section{Creep compliance testing}

Clamps were attached to the ends of the dried films with emory paper placed between the clamp and the sample. This eliminated any extraneous deformation at the clamp. The sample was mounted on a Mettler TMA40 Thermomechanical Analyzer (Mettler Instruments, Hightstown, NJ) and the temperature of the oven brought to about $20^{\circ} \mathrm{C}$ above the sample's softening point. Once thermal equilibrium was obtained the sample was mechanically conditioned by successive loading and unloading steps until a reproducible compliance curve could be observed. Linearity was checked using the method of Ward and Onat (1963) which indicated non-linearity at the highest values of strain. As a result of this testing, final values of strain were always kept below $7 \%$. The stresses that were applied were in the range of $0.9-5 \times 10^{5}$ dynes $/ \mathrm{cm}^{2}$.

The compliance curve for the undiluted Eudragit $\$ 100$ film is illustrated in Fig. 1. A series of tests were performed in uniaxial tension at temperatures ranging from 137 to $157^{\circ} \mathrm{C}$. The recovery was monitored for all the runs and when appropriate, viscous deformation was estimated from the difference between loading and recovery and used to determine the "recoverable" compliance. Fig. 1 indicates a 1000 -fold change in the creep compliance of the film as the test temperature was increased.

The film samples were dissolved in isopropyl alcohol after being mechanically tested and analyzed for plasticizer content. Dibutyl phthalate content was analyzed via UV detection at $280 \mathrm{~nm}$ while PEG content was calculated from the polymer concentration in the solvent. A gel permeation chromatography column was utilized to separate PEG from the polymer and a Waters 410 


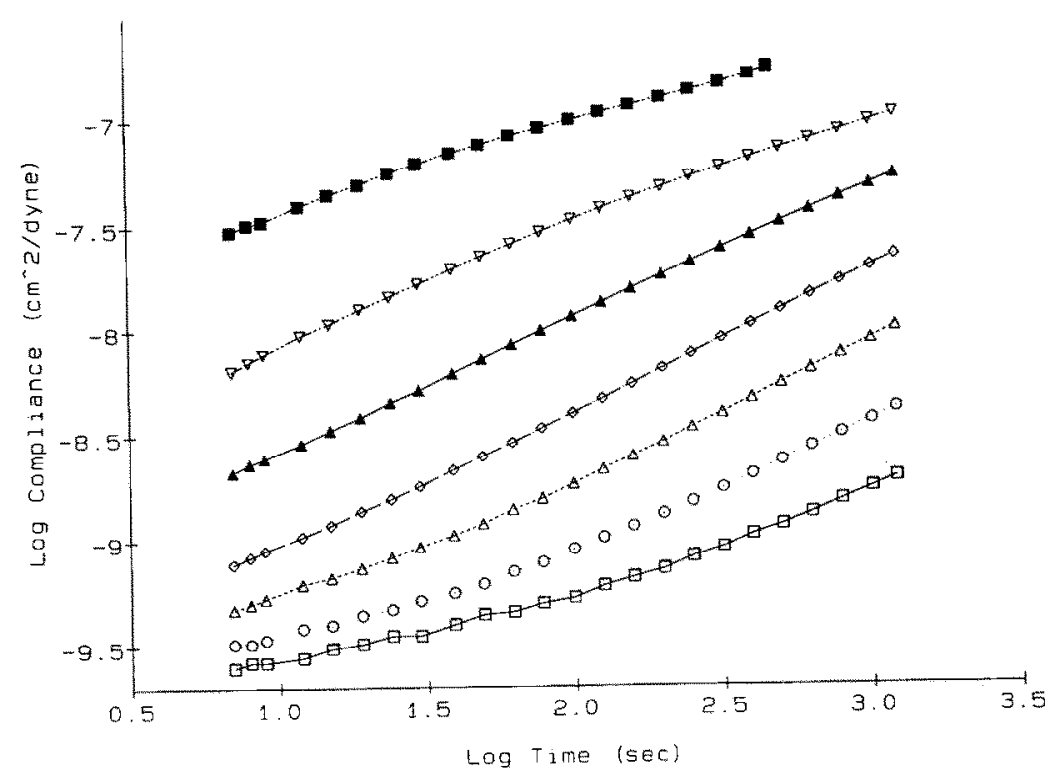

Fig. 1. Creep compliance curves for unplasticized Eudragit S100 at the following temperatures: $\square, 137^{\circ} \mathrm{C} ; 0,139^{\circ} \mathrm{C} ; \Delta, 141^{\circ} \mathrm{C} ; 0$, $143^{\circ} \mathrm{C} ; 1,146^{\circ} \mathrm{C} ; \nabla 150^{\circ} \mathrm{C} ; 157^{\circ} \mathrm{C}$.

Differential Refractometer (Waters \& Associates, Milford, MA) was used to determine the concentration of the polymer.

In all the cases studied, a loss of plasticizer was observed, the extent of which is summarized in Table 1. Plasticizer loss during the compliance experiments would be observed as a reduction in compliance at a specific temperature (Riande et al., 1975). Compliance tests at an intermediate temperature before and after the experiments were

\section{TABLE 1}

Initial and assayed plasticizer concentrations in plasticized Eudragit $\$ 100$

\begin{tabular}{lll}
\hline Plasticizer & $m_{\mathrm{f}}^{*}$ (initial) & $m_{\mathrm{f}}^{*}$ (assayed) \\
\hline PEG & 0.05 & 0.033 \\
PEG & 0.10 & 0.07 \\
PEG & 0.15 & 0.081 \\
PEG & 0.25 & 0.092 \\
DBP & 0.10 & 0.058 \\
DBP & 0.20 & 0.086 \\
DBP & 0.40 & 0.178 \\
DBP & 0.50 & 0.238 \\
\hline
\end{tabular}

* $m_{\uparrow}$ denotes mass fraction of plasticizer. performed revealed that no plasticizer was lost during the experiment. Since the films were dried for approximately 2 weeks, it is likely the plasticizer loss could have taken place during this time. The plasticizer content reported in this paper are the amounts determined by the assay in weight percent.

\section{Results and Discussion}

\section{Temperature reduction}

In order to establish time-temperature equivalence, all the systems tested were assumed to behave as thermorheologically simple materials. This concept, as originally suggested by Schwarzl and Staverman (1952), implies that both recoverable compliance and viscous deformation have the same temperature dependence of response. Time-temperature reduction allows an extension of the time scale for experimental measurement by shifting the compliance curves along the log time axis. This type of shift is only possible if the curves superimpose uniformly and if the temperature dependence of the shift factors, $a_{\mathrm{T}}$, has a form 
TABLE 2

$W L F$ constants and reduction temperatures for plasticized and umplasticized Eudragit $\$ 100$

\begin{tabular}{lllrr}
\hline Plasticizer & $m_{\mathrm{f}}{ }^{*}$ & $T_{\mathrm{r}}\left({ }^{\circ} \mathrm{C}\right)$ & \multicolumn{1}{c}{$C_{1}$} & \multicolumn{1}{c}{$C_{2}$} \\
\hline None & 0 & 139 & 12.54 & 46 \\
PEG & 0.033 & 136 & 21.25 & 86 \\
PEG & 0.07 & 127 & 14.90 & 49 \\
PEG & 0.081 & 124 & 29.54 & 134 \\
PEG & 0.092 & 121 & 7.80 & 24 \\
DBP & 0.058 & 118 & 14.46 & 59 \\
DBP & 0.086 & 115 & 19.32 & 97 \\
DBP & 0.178 & 110 & 17.63 & 102 \\
DBP & 0.238 & 102 & 22.30 & 116 \\
\hline
\end{tabular}

* $m_{f}$ denotes mass fraction of plasticizer.

which can be described by the Williams-LandelFerry (Ferry, 1980c) equation:

$\log a_{\mathrm{T}}=-\mathrm{C}_{1}\left(T-T_{0}\right) /\left(C_{2}+T-T_{0}\right)$

where $C_{1}$ and $C_{2}$ are empirical fitting constants, $T$ is the temperature and $T_{0}$ is the reference temperature for reduction. The WLF parameters for all of the samples tested are presented in Table 2.

A reduced master curve of the data displayed in Fig. 1 is shown in Fig. 2. The reference temper- ature for this reduction was chosen at $139^{\circ} \mathrm{C}$. The polymer exhibits a glass-like response at $\log t / a_{\mathrm{T}}$ $<1.5$. Andrade creep, a phenomena first found in the creep of metals (Andrade, 1962), is observed at $0.5<\log t / a_{\mathrm{T}}<2$. In this region the compliance displays a $t^{1 / 3}$ time dependence. The primary softening dispersion is observed as a large scale change in the compliance of the polymer which occurs between 1.5 and 5 on the reduced time scale. A levelling off to the rubbery plateau starts at $\log t / a_{\mathrm{T}}=4.5$.

The uniformity of the reduced curve satisfies the first criterion for temperature reduction. The second criterion, the description of the temperature dependence of the shift factors with the WLF equation, is shown in Fig. 3. The data points represent the shift factors used to superimpose the curve in Fig. 2 while the line represents the WLF equation with the fitted parameters $C_{1}=12.54$ and $C_{2}=46$. These values are consistent with values determined for a variety of polymers (Ferry, $1980 \mathrm{c}$ ).

The addition of a low molecular weight diluent can change the temperature dependence of response. This point is illustrated in Fig. 3. For the

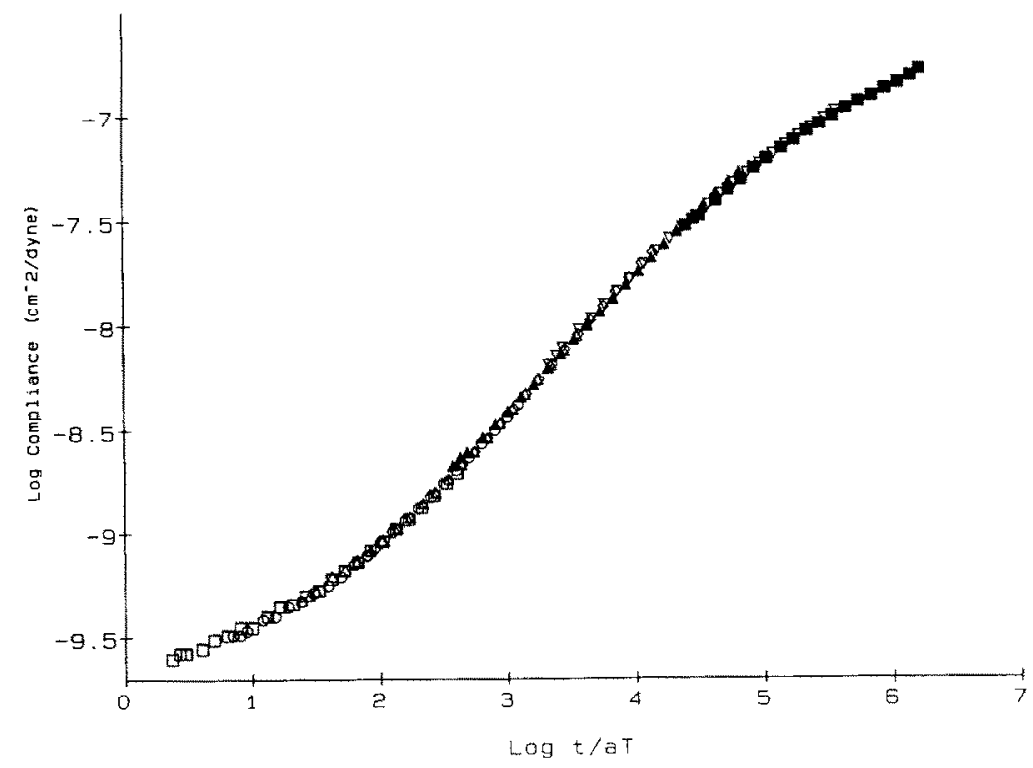

Fig. 2. Master curves of the data presented in Fig. 1 reduced to $139^{\circ} \mathrm{C}$. 


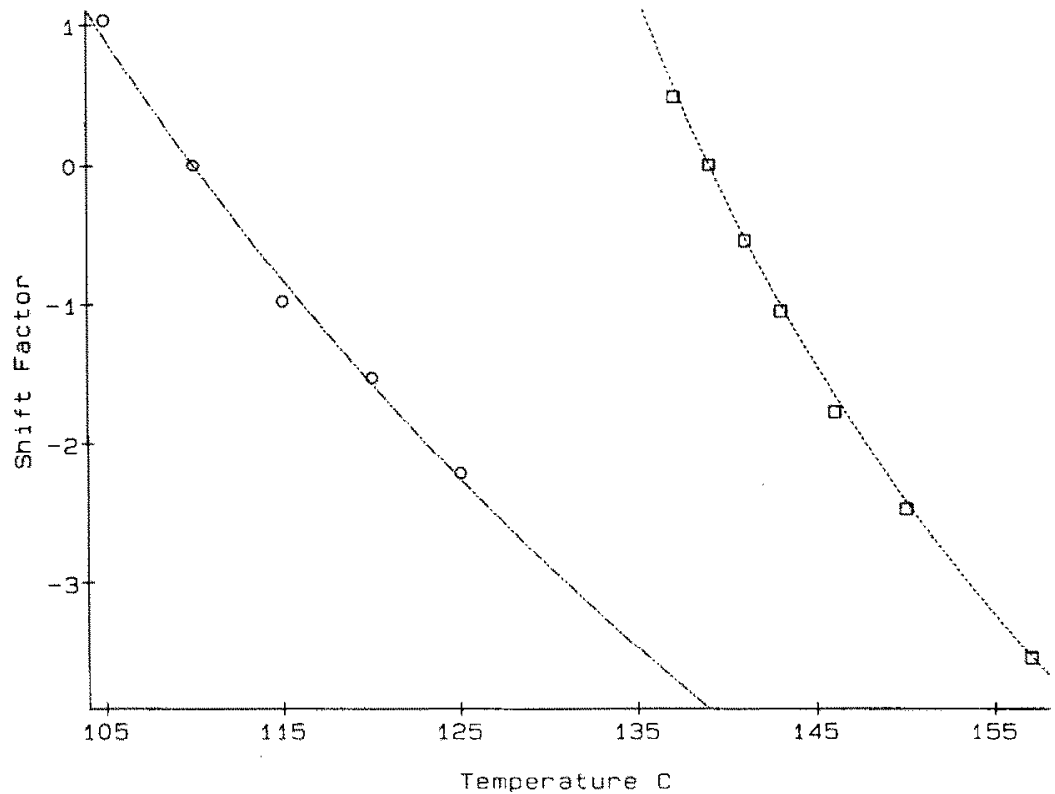

Fig. 3. Shift factors used to reduce data presented in Fig. 2. The lines represent best fits of data to the WLF equation. Symbols used are: $\square$, unplasticized; $0,17 \%$ dibutyl phthalate.

$17 \%$ DBP plasticized system, the mechanical rate of response increases with temperature at a lesser rate than the unplasticized system. The implica- tions of this behavior on the position of the retardation spectra on the reduced time scale is discussed below.

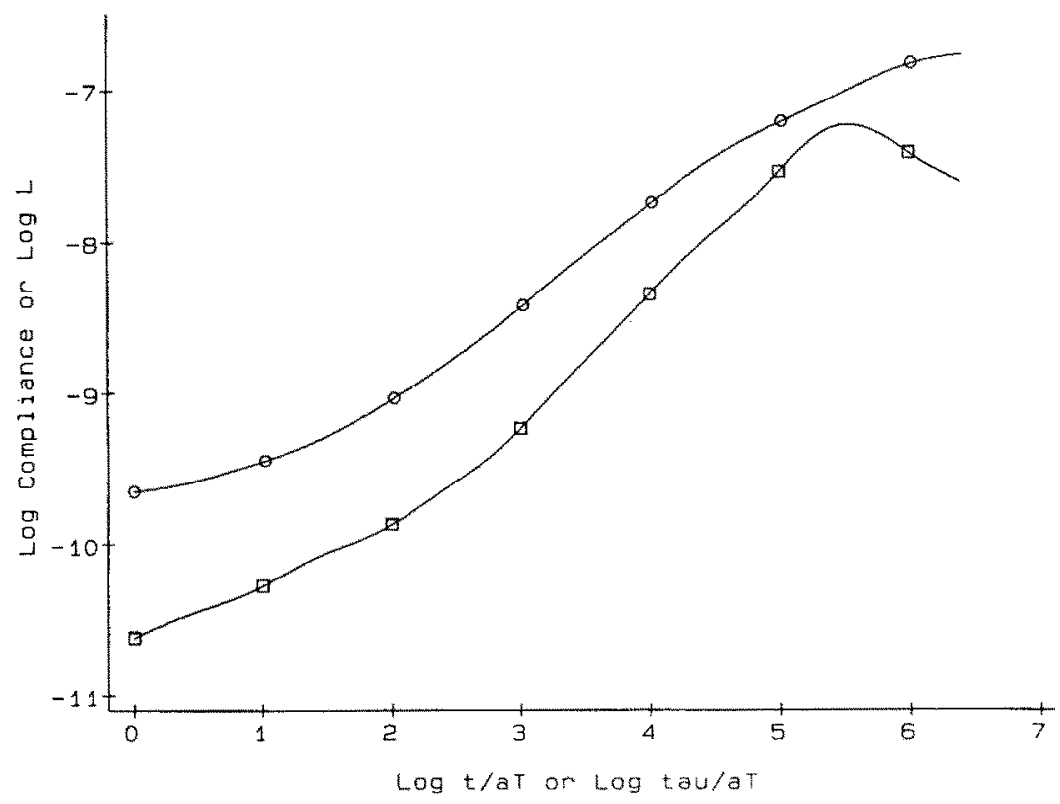

Fig. 4. Retardation spectrum of unplasticized Eudragit $\$ 100$ (C). When curve is placed into Eqn. 2 and integrated, the compliance curve is reproduced $(0)$. 


\section{Retardation spectra}

It is evident from Fig. 2 that the polymer system tested displays a variety of molecular modes of motion which can occur on different time scales. Since individual modes of motion cannot be distinguished, the characterization of intrinsic mechanical behavior will be made on a phenomenological basis. To achieve this the instantaneous compliance is partitioned such that each mode contributes to the overall compliance. A time function similar to a Voight model is assumed for each mode in order for the time-dependent compliance to be represented as a series of weighted exponentials. This analysis, extended to the continuous domain, is represented by Eqn. 2:

$J(t)=J_{\mathrm{g}}+\int_{-\infty}^{\infty} L(\tau)\left(1-\mathrm{e}^{-t / \tau}\right) \mathrm{d} \ln \tau+t / \eta$

Three material parameters arise from this equation: $J_{\mathrm{g}}$, the glassy or instantaneous compliance; $\eta$, the low rate of strain viscosity and $L(\tau)$, the retardation spectrum. The magnitude of the retardation spectrum at a particular retardation time represents the relative importance of the retarda- tion or relaxation process at that time. Since the selection of individual retardation times is of little theoretical significance, a continuous distribution was utilized in this interpretation of the relaxation process.

The retardation spectrum is the material parameter that is used in this study of time-dependent response. The spectrum can be estimated from compliance data using a previously published method (Riande et al., 1975). Fig. 4 details the retardation spectrum of Eudragit S100 along with the reduced master curve from Fig. 2. The peak at $\log \tau / a_{\mathrm{T}}=5.54$ is the region of time where the largest population of mechanisms that contribute to retarded compliance exists at this particular temperature.

\section{Plasticization}

The effect of plasticization on polymeric material behavior has been a focus of research for many years. This is due to the great change in mechanical, thermal and solution properties that are caused by the presence of a low molecular weight diluent. Some of this research has been centered on the magnitude of change in the properties or the efficiency in which a plasticizer could elicit a change.

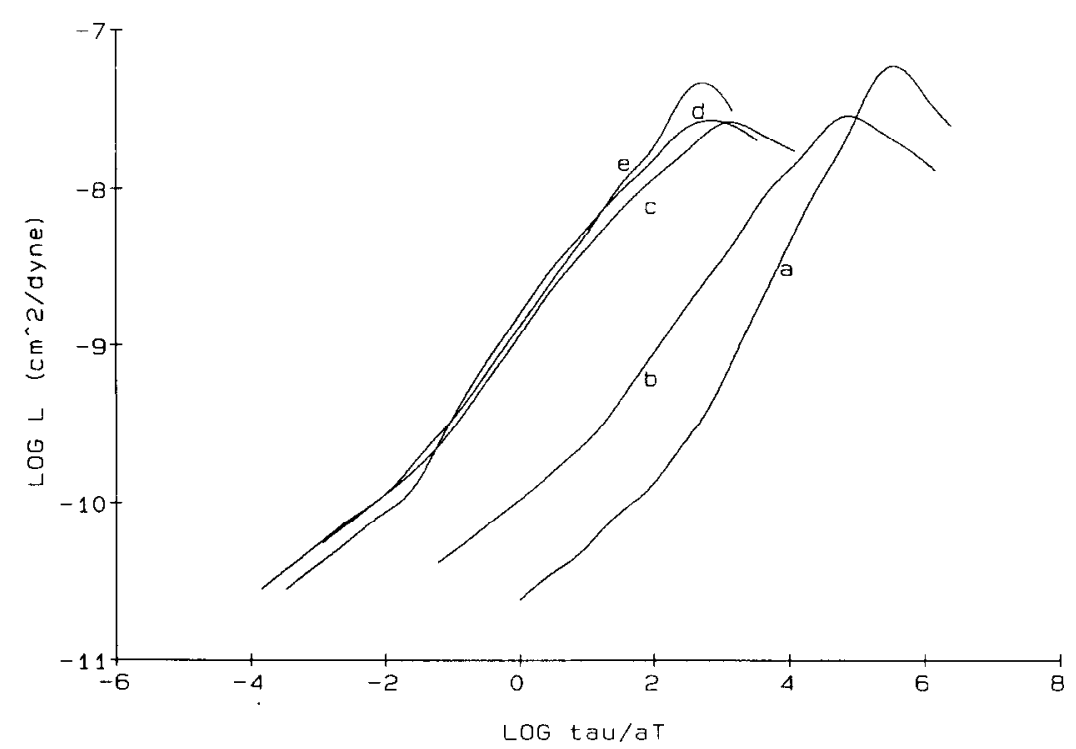

Fig. 5. Corresponding temperature plot of retardation spectra for polyethylene glycol plasticized Eudragit S100. (a) $0 \%$; (b) 3.3\%; (c) $7 \%$; (d) $8.1 \%$; (e) $9.2 \%$. Percentages expressed as weight percent. 
Sakellariou and coworkers (1984) determined the plasticizing efficiency of polyethylene glycol on the controlled release films ethyl cellulose and hydroxypropyl methylcellulose, by evaluating glass transition depression. Although the application of free volume theory to their data was marginally successful, a plasticizing efficiency parameter was calculated using Garfield and Petrie's (1964) theory on free volume additivity. The relation used is shown in Eqn. 3:

$\beta(T)=\alpha_{1}\left(T-T_{\mathrm{g} 1}\right)+\alpha_{2}\left(T-T_{\mathrm{g} 2}\right)$

where $\alpha$ is the thermal expansion coefficient and subscript 1 denotes the plasticizer while subscript 2 denotes the polymer. Sakellariou et al. (1984) and Rowe et al. (1984) found a correlation existed between glass transition depression and $\beta$ for polyethylene glycol plasticized ethyl cellulose and hydroxypropyl methylcellulose but this did not correlate well with dialklyl phthalate plasticized ethyl cellulose. Inserting the values obtained from the literature into Eqn.2: $T_{\mathrm{g}, \mathrm{DBP}}=-97^{\circ} \mathrm{C}, \alpha_{\mathrm{DBP}}$ $=7.8 \times 10^{-4 \circ} \mathrm{C}^{-1} ; T_{\mathrm{g}, \mathrm{PEG}}=-77^{\circ} \mathrm{C}, \quad \alpha_{\mathrm{PEG}}=7.3$ $\times 10^{-4}{ }^{\circ} \mathrm{C}^{-1}$ (Sakellariou et al., 1984; Rowe et al., 1984) the plasticizing effectiveness parameters for PEG and DBP in Eudragit $S$ at $139^{\circ} \mathrm{C}$ were calculated to be 0.158 and 0.184 , respectively.

Fujita (1961) modelled the plasticizing process by predicting the mobility of a penetrant molecule with respect to a fixed polymer network using a free volume analysis. By assuming a linear relationship between the volume fraction of penetrant and the average fractional free volume, the changes in the diffusion coefficient could be estimated. This theory was extended to the mobility of flow units or polymer segments by evaluating viscosity changes due to plasticizer addition. Fujita (1961) arrived at Eqn. 4 with the assumption that the amount of diluent that was added should be sufficiently small.

$$
-v_{1} / \ln \left(D_{T} / D_{0}\right)=f_{2}^{2} / \beta+f_{2} v_{1}
$$

where $f_{2}$ is the fractional free volume of the undiluted polymer at the reference temperature, $v_{1}$ is the volume fraction of the plasticizer and
$D_{\mathrm{T}} / D_{0}$ the ratio of the diffusion coefficient of the solute in the plasticized and unplasticized system.

Plazek and coworkers (1979) evaluated the effect of tricresyl phosphate on the viscoelastic functions of polystyrene. By using the shift of the retardation spectra due to the decreasing concentration of the polymer, $a_{\mathrm{c}}$, the authors analyzed $\beta$ using Fujita's (1961) relation. Shifts of the retardation spectra were determined from the distance between peaks of the dispersion on the log time scale. The shift factor was substituted into Eqn. 4 for the ratio of the diffusion coefficients while the parameter $f_{2}$ was determined from the WLF coefficients of the undiluted polymer and set as a constant in the fitting procedure (Ferry, 1980d).

The analysis outlined by Plazek et al. (1979) was employed on the plasticized systems in this study. The volume fraction of the plasticizer was determined utilizing the densities of the plasticizers and the polymer at $139^{\circ} \mathrm{C}$. The density of the plasticizer at this temperature was estimated using previously published values for thermal expansion (Sakellariou et al., 1984; Rowe et al, 1984) (sp.grav.DBP $=1.047$; sp.grav.PEG $200=$ 1.127 both at $25^{\circ} \mathrm{C}$ ) and that of the polymer was estimated from linear coefficient of expansion data determined with the TMA system (no load, $0.1^{\circ} \mathrm{C} / \mathrm{min}, \quad \alpha=1.78 \times 10^{-4} \mathrm{~cm} / \mathrm{cm} \cdot \mathrm{deg}$, sp.grav. $=1.0855$ at $25^{\circ} \mathrm{C}$ ). The fractional free volume was calculated using the WLF constants (Ferry, 1980c). The plasticization-induced shifts were calculated using a corresponding temperature analysis. Retardation spectra for the plasticized samples were obtained at their reference temperatures and then, assuming thermorheological simplicity, reduced to $139^{\circ} \mathrm{C}$ using the WLF parameters listed in Table 2. The results for PEG 200 are presented in Fig. 5 while those of dibutyl phthalate are in Fig. 6. Both figures reflect the negative shift of the retardation spectra with plasticization.

A plot of $-v_{1} / \ln a_{\mathrm{c}}$ versus $v_{1}$ is shown in Fig. 7. Though the fit is not exact due to the assumptions made in this analysis a definite relationship does exist. The principle differences between the two curves occurs in not only the intercept but also in the slope. This fact implies that a different 


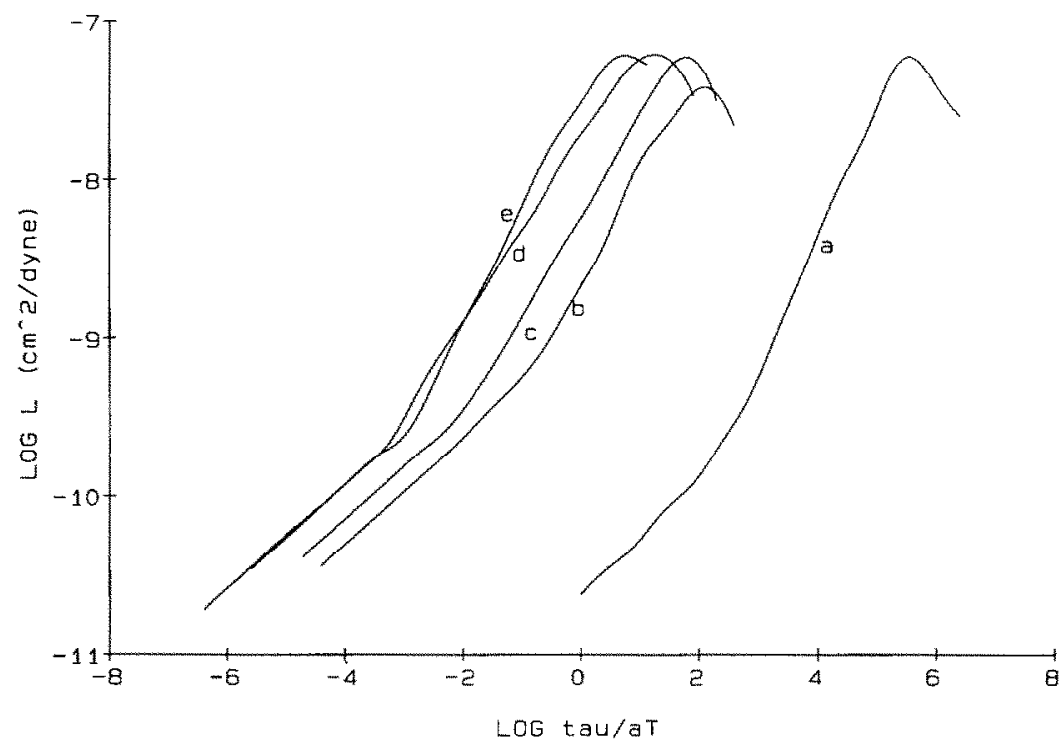

Fig. 6. Corresponding temperature plot of retardation spectra for dibutyl phthalate plasticized Eudragit S100. (a) 0\%; (b) 5.8\%; (c) $8.6 \%$; (d) $17.8 \%$; (e) $23.8 \%$. Percentages expressed as weight percent.

fractional free volume at $T_{\mathrm{r}}$ exists for the undiluted polymer. This clearly violates the assumption of the existence of a single fractional free volume made by Williams et al. (1955) and the assumption of linear free volume additivity comes into question with this significant deviation. Both
Sakellariou et al. (1984) and Plazek et al. (1979) have noted the interaction between polymer and plasticizer may be more complex and this must be reflected in the free volume analysis. Nevertheless, this plot shows a greater intercept for PEG 200 which indicates a lower value of $\beta$. The value of $\beta$

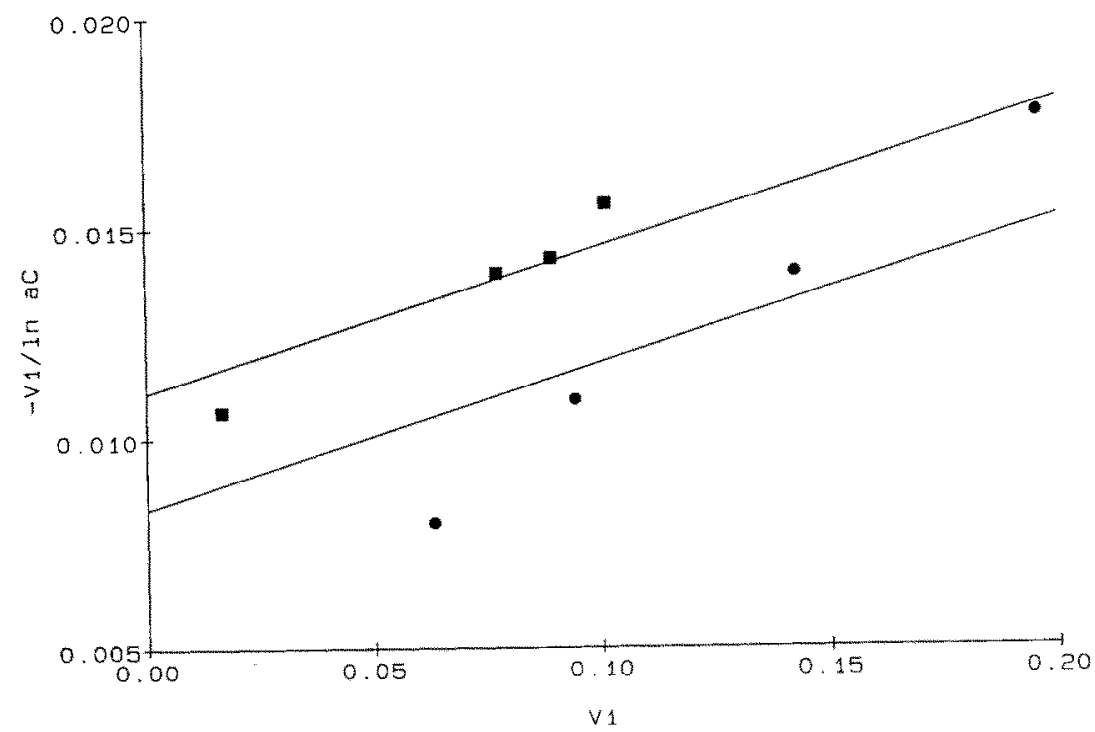

Fig. 7. Plasticizing efficiency determination using Fujita's free volume analysis. Polyethylene glycol (e), dibutyl phthalate (e). 
determined for DBP was 0.144 and that for PEG 200 was 1.08 . Although the numbers are different from those calculated using Eqn. 3, the rank order remains the same. The higher value for dibutyl phthalate indicates that plasticizer's ability to increase segmental mobility in the primary softening region.

A practical application of $\beta$ would be the calculation of a concentration of plasticizer that would result in an equivalent mechanical response as compared to another plasticizer. Using the values of $\beta$ ascertained from the analysis suggested by Plazek et al. (1979), PEG at a volume fraction of 0.15 would elicit an equivalent mechanical response as DBP at a volume fraction of 0.112 . Even though the mechanical response may not be exactly the same, this calculation can be useful in determining which plasticized system has the greater propensity to relax stress.

It should be noted that the shape of the spectrum changes upon plasticization. This is seen most significantly with the $23 \%$ DBP plasticized spectrum in Fig. 6 . The entire primary softening dispersion occurs within 6 logarithmic decades while the spectra for the lower plasticized samples reveals the dispersion region to take place within 4.5 logarithmic decades. Plazek et al. (1979) have observed similar results for polystyrene samples that have a plasticizer concentration greater than $15 \%$.

There are two points to keep in mind when cvaluating a plasticizer's effectiveness at moving the primary softening region. First, the location of the peak of the spectra on the time scale relative to its glass-like response is a direct consequence of the rubbery polymer's ability to respond mechanically, irrespective of the test temperature within the softening region. This was seen above with the apparent expansion of the $23 \%$ DBP spectra as compared to the spectra of the undiluted polymer. Second, the movement of the spectra on the time scale is due solely to the observed temperature dependence of mechanical response. If a plasticizer changes this dependence, for example by accelerating the response, the spectra will appear further away from the undiluted spectra instead of closer if the response was decelerated as was the case for most of the plasticized systems that were studied here. Differences in the rate of change of response can be observed by comparing shift factor-temperature curves. Fig. 3 shows the differences in the curves due to plasticization.

The determination of plasticizing effectiveness via a mechanical analysis is reasonable if the ultimate goal is to understand the mechanical behavior of a system. Movement of the glass transition temperature by itself does not reveal as much information about a film-forming system's ability to relax any stress generated during the drying process although qualitative information can be obtained because it discloses vital information about long range order and conformation. Nevertheless, the glass transition does not describe the mechanical behavior of the film-forming system and insight into this behavior is an important step to a successful formulation.

An analysis of vehicle effects on mechanical response would be the obvious choice for future research. Because of interferences due to solvent loss, a complete characterization of the primary softening dispersion would be difficult experimentally. Qualitative information about plasticization effects could be obtained though by evaluating the rate of response of short-time compliance experiments at a typical processing temperature. Once the effects of various levels of vehicle were quantified at one concentration of plasticizer, comparisons could be made with other concentrations in exactly the same manner as presented in this study by evaluating response at a constant level of vehicle instead of a constant temperature. A plasticizing effectiveness parameter could then be extracted from this analysis. This type of experiment would yield relevant information about the stress relaxation behavior of the polymer-plasticizer-vehicle system.

\section{Conclusion}

The viscoelastic properties of plasticized and unplasticized Eudragit S100 were evaluated in the glass to rubber transition range. Segmental mobility was assessed by estimating intrinsic mechanical response from this data. The effects of plasticization on the mechanical rate of response were 
studied using a corresponding temperature analysis. Results indicate an increase in the rate of response with respect to increasing plasticizer concentration. A free volume analysis was used to calculate plasticizing effectiveness and it has been shown that dibutyl phthalate is more effective at increasing segmental mobility of Eudragit S100 than polyethylene glycol 200 . This approach to measuring the extent of film plasticization may provide a more reliable measure of plasticizer efficiency than glass transition analysis.

\section{Acknowledgements}

This study was supported by an Advanced Predoctoral Fellowship in Pharmaceutics from the Pharmaceutical Manufacturers Assocation Foundation and the Lilly Endowment Fellowship in Pharmacy.

\section{References}

Andrade, E.N. da C., The validity of the $t^{1 / 3}$ law of flow of metals. Philos. Mag., 7 (1962) 2003-2014.

Croll, S.G., The origin of residual internal stress in solvent-cast thermoplastic coatings. J. Appl. Polym. Sci., 23 (1979) 847-858.

Ferry, J.D., Viscoelastic Properties of Polymers, 3rd edn., J. Wiley, New York, 1980a, pp. 486-545.

Ferry, J.D., Viscoelastic Properties of Polymers, 3rd edn., J. Wiley, New York, 1980b, pp. 264-321.

Ferry, J.D., Viscoelastic Properties of Polymers, 3rd edn., J. Wiley, New York, 1980c, pp. 280-291.
Ferry, J.D., Viscoelastic Properties of Polymers, 3rd edn., J. Wiley, New York, 1980d, p. 492.

Fujita, H., Diffusion in polymer-diluent systems. Fortsch. Hochpolym.-Forsch., 3 (1961) 1-47.

Garfield, L.J. and Petrie, S.E., Viscosity and glass-transition behavior of polymer-diluent systems. J. Phys. Chem., 68 (1964) 1750-1754.

Plazek, D.J., Riande, E., Markovitz, H. and Raghupathi, N., Concentration dependence of the viscoelastic properties of polystyrene-tricresyl phosphate solutions. J. Polym. Sci., Polym. Phys. Ed., 17 (1979) 2189-2213.

Riande, E., Markovitz, H., Plazek, D.J. and Raghupathi, N., Viscoelastic behavior of polystyrene-tricresyl phosphate solutions. J. Polym. Sci, Polym. Symp., 50 (1975) 405-430.

Rowe, R.C., Kotaras, A.D. and White, E.F.T., An evaluation of the plasticizing efficiency of the dialkyl phthalates in ethyl cellulose films using the torsional pendulum. Int. $J$. Pharm., 22 (1984) 57-62.

Sakellariou, P., Rowc, R.C. and White, E.F.T., An evalution of the interaction and the plasticizing efficiency of the polyethylene glycols in ethyl cellulose and hydroxypropyl methylcellulose films using the torsional braid pendulum. Int. $J$. Pharm., 31 (1984) 55-63.

Schwarzl, F. and Staverman, A.J., Time-temperature dependence of linear viscoelastic behavior. J. Appl. Phys., 23 (1952) 838-843.

Sinko, C.M. and Amidon, G.L., Viscoelastic properties of Eudragit S/PEG 200 blends. In P.I. Lee and B.A. Leonardt (Eds.), Proceedings of the International Symposium on Controlled Release Bioactive Materials, Vol. 14, 1987, pp. 71-72.

Ward, I.M. and Onat, E.T., Non-linear mechanical behavior of oriented polypropylene. J. Mech. Phys. Solids, 11 (1963) 217-229.

Williams, M.L., Landel, R.F. and Ferry, J.D., The temperature dependence of relaxation mechanisms in amorphous polymers and other glass-forming liquids. J. Am. Chem. Soc., 77 (1955) 3701-3707. 